\title{
The Pin 1 inhibitor juglone attenuates kidney fibrogenesis via Pin 1-independent mechanisms in the unilateral ureteral occlusion model
}

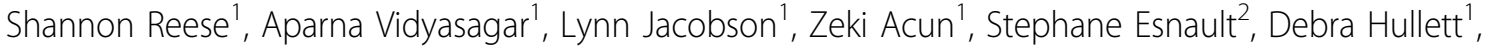 \\ James S Malter ${ }^{2}$, Arjang Djamali ${ }^{{ }^{*}}$
}

\begin{abstract}
Background: Pin 1 is a peptidyl-prolyl isomerase inhibitor related to cyclophilin A and FK506 binding protein (FKBP). Juglone (5-hydroxy-1,4-naphthoquinone) is a natural inhibitor of Pin 1 with anti-inflammatory and antifibrotic properties. We evaluated the role of Pin 1 in renal fibrogenesis by evaluating the effects of juglone on epithelial to mesenchymal transition (EMT) and fibrogenesis in the rat unilateral ureteral obstruction (UUO) model and normal rat tubular epithelial cells (NRK52E).

Results: After 2 weeks of UUO, immunoblot analyses demonstrated that juglone $(0.25$ and $1 \mathrm{mg} / \mathrm{kg} / 24 \mathrm{~h})$ inhibited the deposition of matrix ( $\alpha$-smooth muscle actin (SMA), collagen type III and vimentin) and the activation of signaling pathways involved in fibrogenesis (phospho-smad2) and stress response (phospho-heat shock protein (HSP)27). Juglone also reduced EMT ( $\alpha$-SMA and E-cadherin dual staining) and oxidative stress (Mn superoxide dismutase (SOD) and NAPDH oxidase 2 (Nox-2) dual staining) in the obstructed kidney. There was no difference in Pin 1 levels between treatment and control groups. Pin 1 activity was significantly decreased in obstructed kidneys regardless of treatment status. In vitro, juglone $(1 \mu \mathrm{M})$ significantly decreased $\alpha$-SMA and p-smad levels compared to vehicle.
\end{abstract}

Conclusions: Juglone attenuates fibrogenesis via Pin 1-independent mechanisms in the UUO model. The antifibrotic effects of juglone may result from the inhibition of smad2 and oxidative stress.

\section{Background}

Obstructive nephropathy is a major cause of renal failure, particularly in infants and children $[1,2]$. Urinary tract obstruction and tubular dilatation result in a series of proinflammatory events that ultimately lead to chronic tubulointerstitial fibrosis and kidney failure $[1,2]$. Fibrogenesis starts with the activation of the renin-angiotensin system, tubular apoptosis and macrophage infiltration and is accompanied by the accumulation of interstitial fibroblasts from either proliferation of resident cells or epithelial to mesenchymal transition (EMT) $[1,2]$. The rodent unilateral ureteral obstruction (UUO) model has emerged as an important platform for the study of complex cellular interactions that regulate

\footnotetext{
* Correspondence: axd@medicine.wisc.edu

'Departments of Medicine and Surgery, University of Wisconsin-Madison School of Medicine and Public Health, Madison, WI, USA
}

the development of interstitial inflammation, tubular apoptosis and interstitial fibrosis in this milieu [3]. Evidence suggests that the UUO model is reflective of human kidney disease [3]. Studies examining the mechanisms of fibrogenesis in UUO may therefore result in the development of therapies that will prevent or reverse the structural and functional consequences of obstructive nephropathy [3].

Pin 1 is a cis-trans peptidyl-prolyl isomerase (PPIase) related to cyclophilin A and FK506 binding protein (FKBP) [4,5]. Pin 1 modulates cytokine expression by activated T cells and eosinophils and participates in $\mathrm{T}$ cell and eosinophil apoptotic decisions both in vitro and in vivo [5]. In addition, Pin 1 blockade attenuates transforming growth factor $\beta 1$ (TGF $\beta$ 1) and granulocytemacrophage colony-stimulating factor (GM-CSF) production and inflammation in experimental models of allergic lung fibrosis $[4,6]$. We therefore hypothesized 
that Pin 1 plays a role in kidney fibrogenesis and tested this hypothesis in vivo using the rodent UUO model and in vitro using normal rat proximal tubular epithelial cells (NRK52E). We used juglone (5-hydroxy-1,4naphthoquinone) a natural inhibitor of Pin 1 to characterize the effects of Pin 1 inhibition on fibrogenesis.

\section{Results}

\section{Juglone reduced fibrogenesis after UUO}

Male Lewis rats (3 months old) underwent UUO of the left kidney for 2 weeks. There were three groups receiving vehicle, juglone $0.25 \mathrm{mg} / \mathrm{kg} /$ day or juglone $1 \mathrm{mg} / \mathrm{kg} /$ day for 2 weeks starting the day of surgery. There was no animal death associated with treatment. Treated animals had a $10 \%$ weight loss in the first week after surgery, which resolved by the end of week 2 . Immunoblot analyses for Pin 1, biomarkers of matrix remodeling $(\alpha-$ smooth muscle actin (SMA), collagen type III and vimentin) and signaling pathways involved in fibrogenesis (phospho-smad2) and stress response (phospho-heat shock protein (HSP)27) demonstrated that juglone therapy decreased $\alpha$-SMA, collagen type III, vimentin, psmad2 and p-HSP27 levels (Figure 1). There was no difference in Pin 1 levels between treatment and control groups suggesting that juglone inhibits fibrogenesis independently of Pin 1 levels in the UUO model.

\section{Juglone and UUO had similar inhibitory effects on Pin 1} activity

We next examined Pin 1 activity in unobstructed and obstructed kidneys in control or juglone-treated rats. These analyses would help us determine whether the antifibrotic properties of juglone resulted from Pin 1 blockade. The studies demonstrated that juglone effectively inhibited Pin 1 activity in unobstructed right kidneys (Figure 2). However, Pin 1 activity was significantly decreased in left obstructed kidneys regardless of treatment status (Figure 2). Pin 1 activity in obstructed kidneys was reduced to the same level as in unobstructed kidneys treated with juglone. In aggregate, these studies suggest that the antifibrotic effects of juglone are independent from Pin 1 blockade during UUO.

Juglone reduced EMT and oxidative stress after UUO

To further define the effects of juglone on fibrogenesis, we assessed EMT using double-staining immunohistochemical analyses for E-cadherin (pink, epithelial marker) and $\alpha$-SMA (brown, mesenchymal marker) in normal right and UUO left kidneys in control or juglone-treated rats (Figure 3a-c). These studies showed pink basolateral E-cadherin staining in distal tubules of control kidneys (Figure 3a). UUO resulted in downregulation of E-cadherin (no basolateral staining) and greater brown interstitial staining for $\alpha$-SMA (Figure $3 b$ ). Treatment with juglone preserved distal E-cadherin

\section{(a) Immunoblots of UUO kidneys}

\section{(b) Average protein levels}
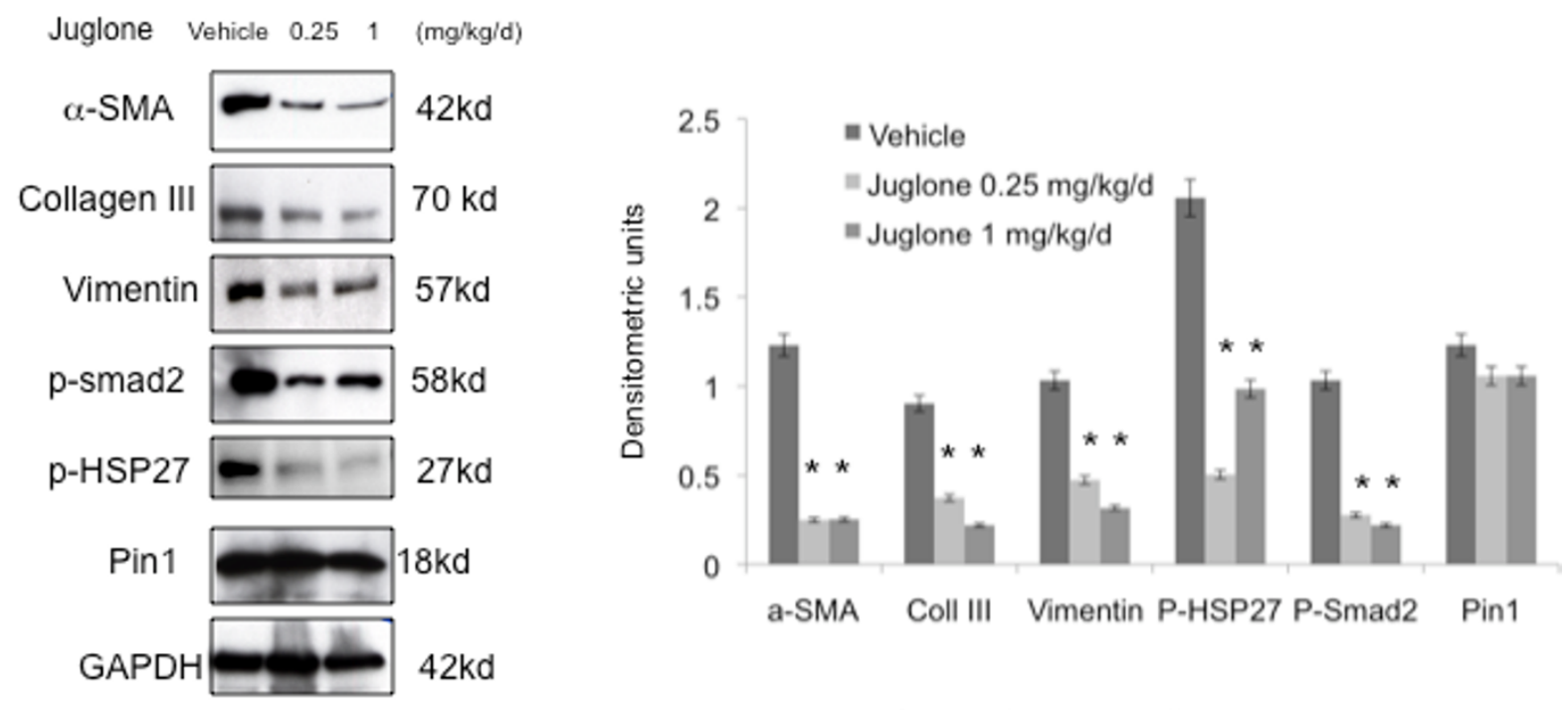

${ }^{\star} \mathrm{p}<0.05$ compared to no treatment

Figure 1 Juglone reduced fibrogenesis after unilateral ureteral obstruction (UUO). Male Lewis rats ( 3 months old) underwent UUO of the left kidney for 2 weeks. There were three groups receiving vehicle or juglone $(0.25 \mathrm{mg} / \mathrm{kg} /$ day or $1 \mathrm{mg} / \mathrm{kg} /$ day $)$ for 2 weeks starting the day of surgery. (a) Immunoblot analyses of left kidney from control or juglone-treated rats for the proteins shown along the left. (b) Multiple ( $n=3$ ) immunoblots were quantitated and signals expressed as arbitrary units after normalization to glyceraldehyde 3-phosphate dehydrogenase (GAPDH) signal. 


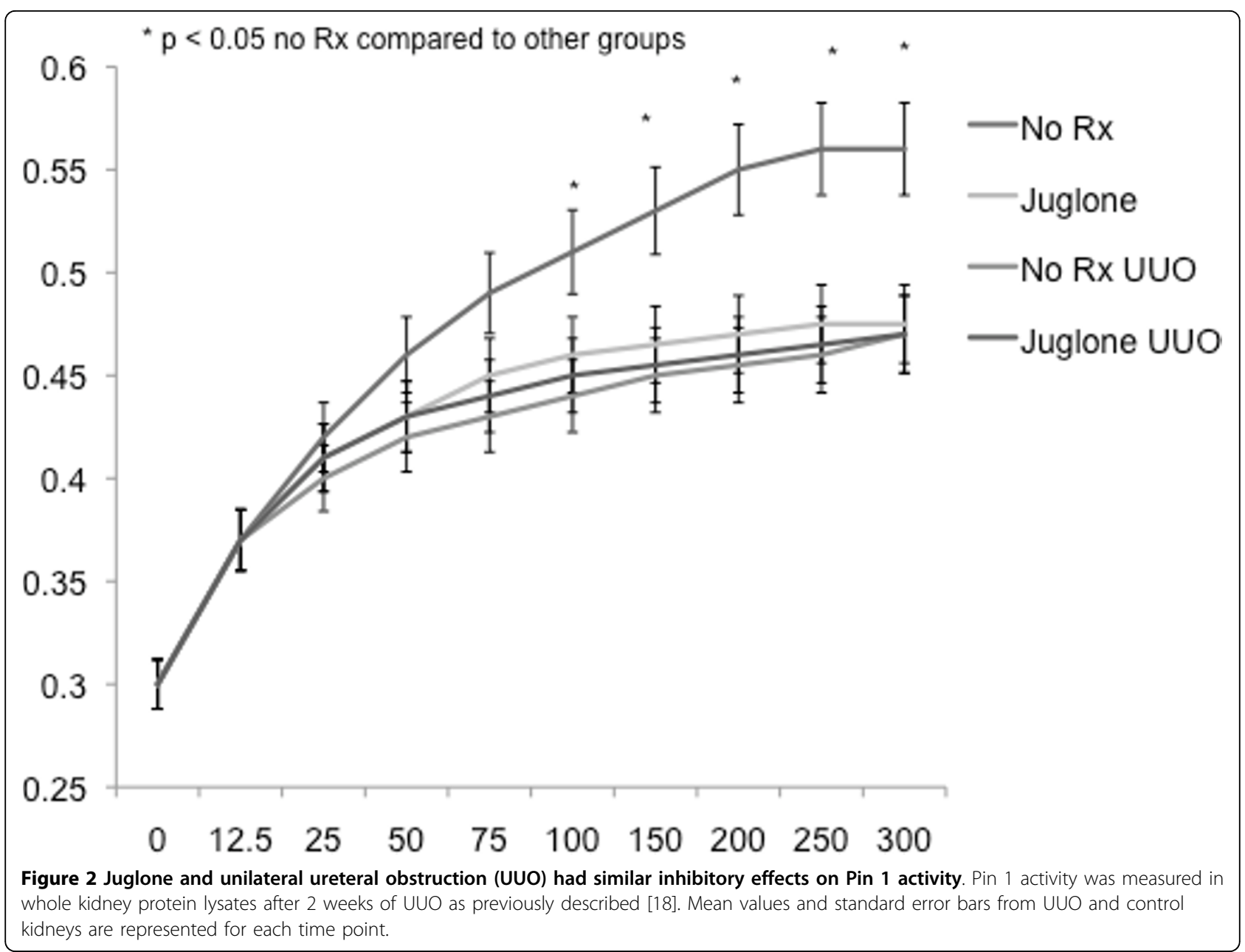

expression and decreased interstitial $\alpha$-SMA levels (Figure 3c), suggesting that juglone may attenuate EMT during UUO. Aggregate semiquantitative scoring is presented as a bar graph in the right panel (Figure 3).

We evaluated oxidative stress using dual-staining analyses for antioxidant (Mn superoxide dismutase (SOD)) and pro-oxidant (NAPDH oxidase 2 (Nox-2)) enzymes (Figure $3 \mathrm{~d}-\mathrm{f}$ ). MnSOD is a superoxide scavenger while Nox-2 is one of the key generators of superoxide in the kidney [7]. Normal kidneys showed strong tubular MnSOD staining and rare patchy areas of monocytic infiltration positive for Nox-2 (Figure 3d). UUO resulted in reduced tubular MnSOD and increased tubulointerstitial Nox-2 (Figure 3e) consistent with significant monocyte/macrophage infiltration and a pro-oxidant milieu. Juglone attenuated these changes suggesting that treatment decreases oxidative stress (Figure $3 \mathrm{f}$ ). Consistent with these findings, dihydroethidine staining for superoxide anion was significantly decreased with juglone compared to vehicle (Figure 3g, h). Aggregate semiquantitative scoring is presented as a bar graph in the right panel (Figure 3).

The effects of juglone on fibrogenesis may be mediated by smad2

To determine whether juglone inhibited TGF 1 activity we evaluated phospho-smad2 activity in normal kidneys compared to obstructed kidneys treated or not with juglone (Figure 4). These studies demonstrated that nuclear p-smad2 was significantly increased after UUO and that juglone prevented nuclear p-smad2 activity. Lastly, we evaluated the effects of juglone on $\alpha$-SMA and activated smad2 ( $\mathrm{p}$-smad2) levels in proximal tubular epithelial cells. Juglone $(1 \mu \mathrm{M})$ significantly reduced $\alpha$-SMA and p-smad2 levels, consistent with our in vivo studies and suggesting that juglone may inhibit smad2 phosphorylation and activation in tubular epithelial cells (Figure 5).

\section{Discussion}

In the present work we demonstrate that juglone, a naturally occurring Pin1 isomerase inhibitor, attenuates 


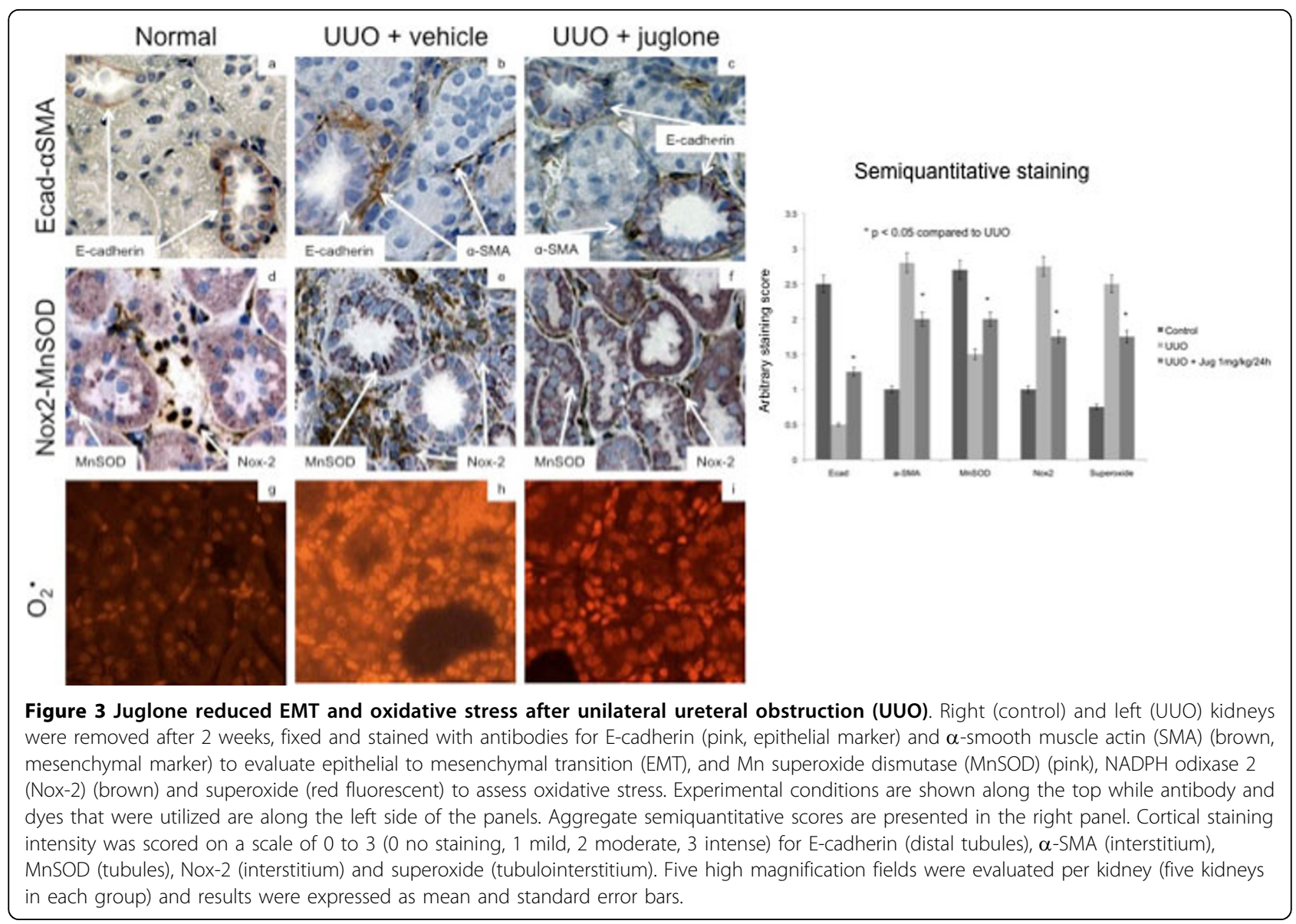

fibrogenesis in kidneys undergoing obstructive injury. This effect appears to be Pin 1 independent as PPIase activity was unchanged in the UUO left kidney between control and juglone-treated rats. Our studies further suggest that the antifibrotic effects of juglone result from the inhibition of smad2 phosphorylation and oxidative stress.

Juglone is a napthaquinone found in the leaves, roots and bark of plants from the walnut family. It is toxic to the growth of non-walnut plants and likely exerts its effect by inhibiting peptidyl-prolyl isomerases found in plants. Juglone has differential effects on cell cycling and metabolism depending on the species, organ and drug concentrations [8]. In F344 rats, high concentrations of juglone-derived radioactivity were found in the kidney after oral, intravenous and subcutaneous dosing [9]. The accumulation in the kidney was attributed to covalent binding of juglone and its metabolites to cytosolic proteins and suggests that the kidney may be a potential treatment target for juglone. In support of this hypothesis, juglone increased the activities of phase II detoxification enzymes quinone reductase and glutathione transferase in the kidney of Sprague-Dawley rats suggesting a role for this compound to protect animals against toxin-induced kidney injury [10].

Our results are in agreement with previous observations addressing the antifibrotic and anti-inflammatory characteristics of juglone in experimental models of lung injury $[6,11]$. These studies demonstrated that juglone therapy selectively inhibited eosinophilic and lymphocytic inflammation in rats undergoing experimental allergic lung fibrosis [6] and lung allograft rejection [11]. Juglone reduced eosinophilic pulmonary inflammation, TGF $\beta$ 1, collagen expression and airway remodeling in rats undergoing allergic lung fibrosis [6]. Similarly, juglone treatment prevented the acute and chronic rejection of major histocompatibility complex (MHC)-mismatched, orthotopic rat lung transplants by reducing the expression of proinflammatory interferon (IFN) $\gamma$ and CXC chemokine ligand (CXCL) 10 cytokines [11]. In these studies, combined transcriptional and post-transcriptional blockade of cytokine expression with cyclosporine A and the juglone was synergistic [11]. Our findings extend these data by demonstrating that juglone also attenuates inflammation in a macrophage-driven kidney injury model [1-3]. 

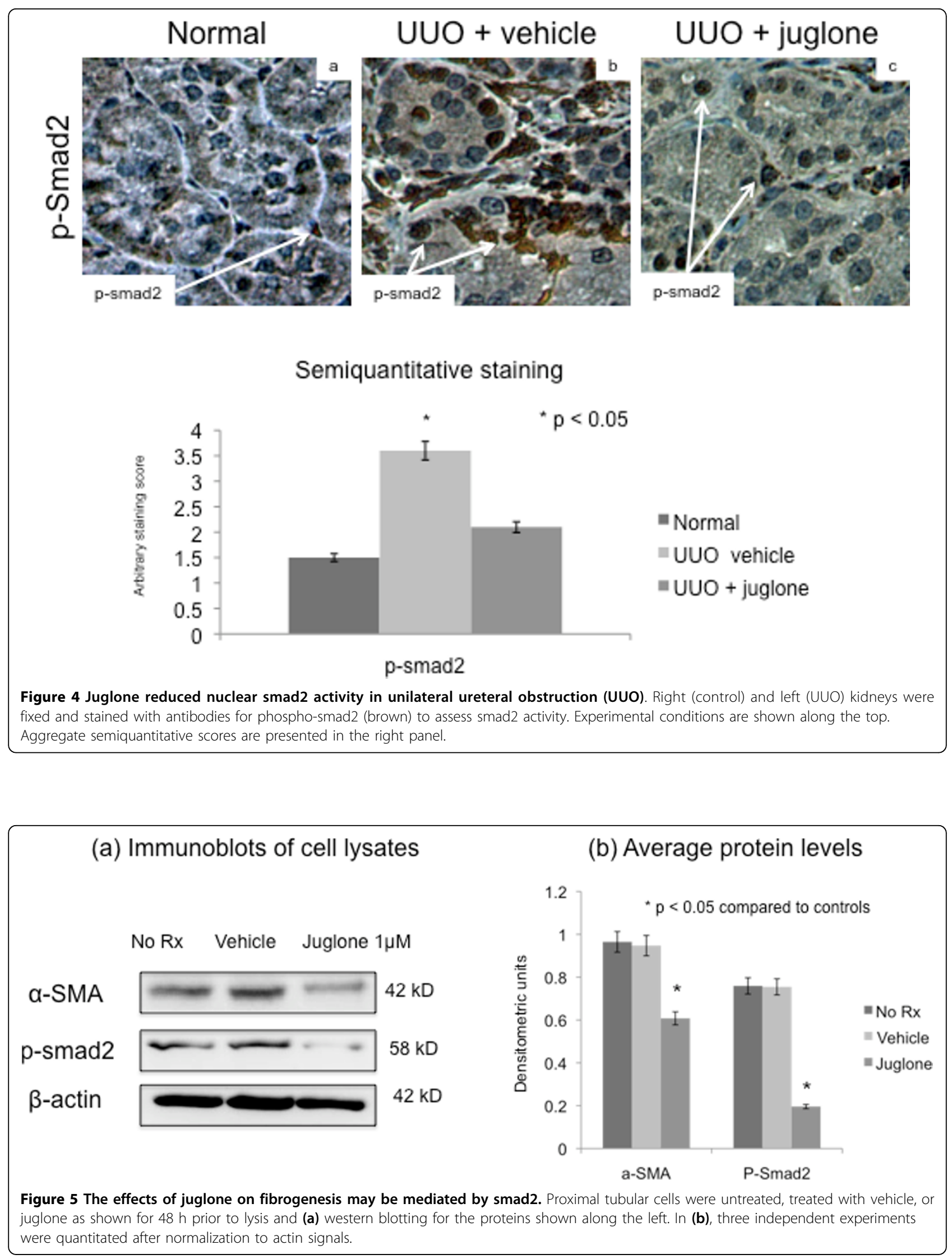
Interestingly, the anti-inflammatory effects of juglone were not dependent on Pin 1 blockade in the UUO model. Rather, they were associated with the inhibition of oxidative stress and smad2 phosphorylation. Juglone may have either pro or antioxidant characteristics depending on the milieu and drug concentrations [8]. While its use can result in the generation of reactive oxygen species in Caenorhabditis elegans [12], juglone is a potent antioxidant in human cortical neurons [13]. Juglone treatment prevents oxidative and heat stressinduced dephosphorylation of Tau (an important step in the pathogenesis of Alzheimer's disease) in primary brain cortical cultures [13]. Oxidative stress is a common injury pathway involved in kidney fibrogenesis $[14,15]$. We recently demonstrated that specific inhibitors of Nox (the primary generator of superoxide anion in the kidney) decreased fibrogenesis in kidney allografts by decreasing fibronectin and phospho-smad2 and increasing E-cadherin levels [7]. We have now demonstrated that juglone improves the oxidative stress balance in the UUO model by downregulating Nox-2 and superoxide anion while increasing tubular MnSOD levels. In addition, we have shown that juglone inhibits the phosphorylation of smad2 an important redox-sensitive, profibrotic signaling molecule in the kidney $[7,16,17]$. Although it is unclear whether the inhibition of smad2 phosphorylation was a direct effect or a downstream event secondary to juglone's antioxidant characteristics in our model, smad2 inhibition has been successful in experimental studies of native and transplants kidney fibrosis $[7,16,17]$.

\section{Conclusions}

In summary, these studies demonstrate that juglone attenuates fibrogenesis in kidneys undergoing obstructive injury via Pin 1-independent mechanisms. The antifibrotic effects of juglone may result from the inhibition of inflammation and more specifically smad 2 and oxidative stress. Future studies are needed to determine the cellular and molecular mechanisms that regulate the inhibitory effects of juglone on smad and Nox molecules.

\section{Methods}

\section{Animals}

Adult (9 to 11 weeks old) male Lewis rats were purchased from Harlan Teklad (Madison, WI, USA). Animals were housed in the animal care facility at the William Middleton Veterans Affairs Hospital (VAH) in Madison, WI, USA, and the procedures were performed in accordance with the animal care policies at the VAH and the University of Wisconsin. The UUO procedure was performed under general anesthesia with isoflurane as described previously [14]. Briefly, the left ureter was ligated with 6-0 silk at two points and then severed between the ligatures to prevent retrograde urinary tract infection. Control animals underwent surgery and received vehicle $(10 \%$ ethanol) $(n=5)$. Juglone was administered intraperitoneally for 14 days at $0.25(\mathrm{n}=$ $5)$ and $1 \mathrm{mg} / \mathrm{kg} / 24 \mathrm{~h}$ diluted in $1 \mathrm{ml}$ of vehicle $(\mathrm{n}=5)$. Animals were killed after 2 weeks by exsanguination through cardiac puncture under general anesthesia. Both kidneys were harvested and sectioned longitudinally in half. Half was snap frozen immediately and used for immunoblot analysis and the other half was formalin fixed and paraffin embedded for immunohistochemical analyses. The right kidney served as the control to the left obstructed kidney.

\section{Immunoblotting}

Western blotting was performed on protein lysates obtained from whole kidney tissue or cell lysates as described previously [14]. Briefly, after separation by SDS-PAGE (10\% to $20 \%$ gradient PAGE, Bio-Rad, Hercules, CA, USA) proteins were transferred electrophoretically $(100 \mathrm{~V}, 30 \mathrm{~min})$ to nitrocellulose membranes (Bio-Rad) that were then blocked with a solution containing 5\% non-fat milk, $50 \mathrm{mM}$ Tris, $\mathrm{HCl}, \mathrm{pH} 7.4$, $\mathrm{NaCl} 150$ mM, Tween 20 0.05\% (TBS-Tween) overnight at $4^{\circ} \mathrm{C}$. Membranes were incubated the next day with antibodies against $\alpha$-SMA $\left(2,000^{-1}\right)$, Vimentin $\left(100^{-1}\right)$, collagen type III $\left(100^{-1}\right)$, phospho-HSP27 $\left(0.25^{-1}\right)$, phospho-smad2 $\left(2,500^{-1}\right)$, Pin $1\left(200^{-1}\right)$, and GAPDH $(1: 5,000)$. Binding of primary antibodies was followed by incubation for $1 \mathrm{~h}$ at room temperature with a secondary horseradish peroxidase (HRP)-conjugated IgG in 1\% non-fat milk. Signals were visualized by enhanced chemiluminescence signals captured on x-ray films. Data was normalized to GAPDH. Densitometry was performed using the NIH Image J software http://rsbweb. nih.gov/ij/.

\section{Immunohistochemical analyses}

A portion of the kidney tissue was excised promptly after the animals were killed. It was immediately placed in $10 \%$ neutral-buffered formalin. Tissue was fixed overnight in formalin and processed for paraffin embedding following standard protocols and then sectioned for antibody staining. Double staining of E-cadherin/ $\alpha-S M A$ and Nox-2/MnSOD was performed after sections were deparaffinized and hydrated. Heat-induced antigen retrieval was performed using a $5 \mathrm{mM}$ ethylenediaminetetra-acetic acid (EDTA) solution $(\mathrm{pH}=8.0)$ and a 10 $\mathrm{mM}$ citrate solution $(\mathrm{pH}=6.0)$, respectively, at $25 \mathrm{psi}$ for $2 \mathrm{~min}$ in a decloaking chamber. Non-specific staining was blocked using Sniper (Biocare Medical, Concord, CA, USA) for $9 \mathrm{~min}$. Slides were incubated overnight with p-smad2 $\left(500^{-1}\right)$, E-cadherin $\left(50^{-1}\right)$ or Nox-2 $\left(50^{-1}\right)$ then washed and incubated with $3 \%$ hydrogen peroxide for $30 \mathrm{~min}$. MACH $2 \mathrm{HRP}$ polymer 
detection system (Biocare Medical, Concord, CA, USA) and 3,3'-diaminobenzidine (DAB) substrate were used to tag and stain the first primary antibody brown. Slides were then incubated with the second primary antibody $\alpha$-SMA $\left(50,000^{-1}\right)$ or $\operatorname{MnSOD}\left(5,000^{-1}\right)$ at room temp for $1 \mathrm{~h}$. MACH $2 \mathrm{HRP}$ polymer detection system and VIP substrate (Vector Laboratories, Burlingame, CA, USA) were used to tag and stain the second primary antibody purple. Tissue sections were washed in distilled water, counterstained with hematoxylin, dehydrated through an ethanol series and mounted with cover slips. Harris hematoxylin and 1\% alcohol eosin were used to assess overall kidney injury and morphology. A total of $25 \mu \mathrm{M}$ dihydroethidine dye (Molecular Probes, Carlsbad, CA, USA) was used for superoxide anion staining according to the manufacturer's recommendations.

Cortical staining intensity was scored on a scale of 0 to 3 (0 no staining, 1 mild, 2 moderate, 3 intense) for Ecadherin (distal tubules), $\alpha$-SMA (interstitium), MnSOD (tubules), Nox-2 (interstitium), p-smad2 and superoxide (tubulointerstitium). Five high magnification fields were evaluated per kidney and results were expressed as mean and standard error bars.

\section{Pin 1 activity assay}

Pin 1 activity was measured in whole kidney protein lysates as described previously [18]. Briefly, tissue lysates were prepared by five freeze-thaw cycles in a buffer containing $50 \mathrm{mM}$ 4-(2-hydroxyethyl)-1-piperazineethanesulfonic acid (HEPES) and $100 \mathrm{mM} \mathrm{NaCl}(\mathrm{pH} \mathrm{7.0)}$. Total protein $(10 \mu \mathrm{g})$ in $10 \mu \mathrm{l}$ was mixed with $70 \mu \mathrm{l}$ of the HEPES/NaCl buffer supplemented with $2 \mathrm{mM}$ dithiothreitol (DTT) and $0.04 \mathrm{mg} / \mathrm{ml}$ bovine serum albumin (BSA). Then, $5 \mu \mathrm{l}$ of chymotrypsin $(60 \mathrm{mg} / \mathrm{ml}$ in $0.001 \mathrm{~N} \mathrm{HCl}$ ) was added and thoroughly mixed. Finally, $5 \mu \mathrm{l}$ of the substrate Suc-AEPF-pNa (provided by Peptides International, Louisville, KY, USA) dissolved in dimethyl sulfoxide (DMSO) and prepared at $100 \mu \mathrm{g} /$ $\mathrm{ml}$ in $480 \mathrm{mM} \mathrm{LiCl} /$ trifluoroethanol was added. The absorption at $390 \mathrm{nM}$, which detects the formation of free p-nitroanilide (pNA), was monitored using a Beckman Coulter

DU 800 spectrophotometer (Brea, CA, USA). All of the reagents and materials were kept at $4^{\circ} \mathrm{C}$ during the procedure. Mean values and standard error bars from UUO and control kidneys are represented for each time point.

\section{Juglone in vitro experiments}

Normal rat kidney proximal epithelial cells (NRK52E) were obtained from the American Type Culture Collection (ATCC, Rockwell, MD, USA) and maintained at $37^{\circ}$ $\mathrm{C}$ in a humidified atmosphere containing $5 \% \mathrm{CO}_{2}$. Cells were seeded at $2.5 \times 10^{5}$ cells per well into six-well culture plates in Dulbecco modified Eagle medium (DMEM; high glucose) containing 5\% heat inactivated fetal bovine serum (FBS), $44 \mathrm{mM} \mathrm{NaHCO} 3,5,000 \mathrm{IU}$ penicillin and $5,000 \mu \mathrm{g} / \mathrm{ml}$ streptomycin (Cellgro, VA, USA). At $80 \%$ confluency, media was changed to serum free DMEM supplemented with $0.1 \%$ BSA for $12 \mathrm{~h}$ to arrest growth and synchronize cell activity. Cells were treated with juglone $(1 \mu \mathrm{M})$ or vehicle $(0.01 \%$ ethanol) for $48 \mathrm{~h}$. Studies were performed in triplicates. Western blots for $\alpha$-SMA, phospho-smad 2 and $\beta$-actin were performed as described above.

\section{Statistical analysis}

The Student $t$ test and the non-parametric Mann-Whitney rank sum test (Sigma Stat Software, Jandel Scientific, Chicago, IL, USA) were utilized when appropriate to compare differences in Pin 1 activity and gene and protein expression between groups. $P$ values $\leq 0.05$ were considered significant.

\section{Acknowledgements}

Parts of this work were supported by an NIH grant DK 067981 and the ASNAST John Merrill Award (AD) and NIH grants R01 HL087950, P01 HL088594 and P30 HD03352 (JSM)

\section{Author details}

'Departments of Medicine and Surgery, University of Wisconsin-Madison School of Medicine and Public Health, Madison, WI, USA. ${ }^{2}$ Department of Pathology and Laboratory Medicine, University of Wisconsin-Madison School of Medicine and Public Health, Madison, WI, USA

\section{Authors' contributions}

$A D$ and JSM developed the concept and design of the study. SR, AV, L, ZA and SE contributed to data acquisition and analyses. SR, SE, DH, JM and AD contributed to the writing of the manuscript and all the authors approved the final manuscript.

\section{Competing interests}

The authors declare that they have no competing interests.

\section{Received: 12 September 2009}

Accepted: 4 January 2010 Published: 4 January 2010

\section{References}

1. Bascands JL, Schanstra JP: Obstructive nephropathy: Insights from genetically engineered animals. Kidney Int 2005, 68:925-937.

2. Chevalier RL: Obstructive nephropathy: towards biomarker discovery and gene therapy. Nat Clin Pract Nephrol 2006, 2:157-168.

3. Klahr S, Morrissey J: Obstructive nephropathy and renal fibrosis. Am J Physiol Renal Physiol 2002, 283:F861-F875.

4. Esnault S, Rosenthal LA, Shen ZJ, Sedgwick JB, Szakaly RJ, Sorkness RL, Malter JS: A critical role for Pin1 in allergic pulmonary eosinophilia in rats. J Allergy Clin Immunol 2007, 120:1082-1088.

5. Esnault $\mathrm{S}$, Shen ZJ, Malter JS: Pinning down signaling in the immune system: the role of the peptidyl-prolyl isomerase Pin 1 in immune cell function. Crit Rev Immunol 2008, 28:45-60.

6. Shen ZJ, Esnault S, Rosenthal LA, Szakaly RJ, Sorkness RL, Westmark PR, Sandor M, Malter JS: Pin1 regulates TGF-beta1 production by activated human and murine eosinophils and contributes to allergic lung fibrosis. J Clin Invest 2008, 118:479-490.

7. Djamali A, Vidyasagar A, Adulla M, Hullett $D$, Reese $S$ : Nox-2 is a modulator of fibrogenesis in kidney allografts. Am J Transplant 2009, 9:74-82.

8. Chobot V, Hadacek F: Milieu-dependent pro- and antioxidant activity of juglone may explain linear and nonlinear effects on seedling development. J Chem Ecol 2009, 35:383-390.

9. Chen $L$, Lebetkin EH, Burka LT: Metabolism and disposition of juglone in male F344 rats. Xenobiotica 2005, 35:1019-1034. 
10. Munday R, Munday CM: Induction of quinone reductase and glutathione transferase in rat tissues by juglone and plumbagin. Planta Med 2000, 66:399-402.

11. Esnault S, Braun RK, Shen ZJ, Xiang Z, Heninger E, Love RB, Sandor M, Malter JS: Pin1 modulates the type 1 immune response. PLOS ONE 2007, 2:e226.

12. de Castro E, Hegi de Castro S, Johnson TE: Isolation of long-lived mutants in Caenorhabditis elegans using selection for resistance to juglone. Free Radic Biol Med 2004, 37:139-145.

13. Galas MC, Dourlen P, Bégard S, Ando K, Blum D, Hamdane M, Buée L: The peptidylprolyl cis/trans-isomerase Pin1 modulates stress-induced dephosphorylation of Tau in neurons. Implication in a pathological mechanism related to Alzheimer disease. J Biol Chem 2006, 281:1929619304.

14. Vidyasagar A, Reese $S$, Acun Z, Hullett D, Djamali A: HSP27 is involved in the pathogenesis of kidney tubulointerstitial fibrosis. Am J Physiol Renal Physiol 2008, 295:F707-716.

15. Djamali A: Oxidative stress as a common pathway to chronic tubulointerstitial injury in kidney allografts. Am J Physiol Renal Physiol 2007, 293:F445-455.

16. Li JH, Zhu HJ, Huang XR, Lai KN, Johnson RJ, Lan HY: Smad7 inhibits fibrotic effect of TGF-beta on renal tubular epithelial cells by blocking Smad2 activation. J Am Soc Nephrol 2002, 13:1464-1472.

17. Rhyu DY, Yang Y, Ha H, Lee GT, Song JS, Uh ST, Lee HB: Role of reactive oxygen species in TGF-beta1-induced mitogen-activated protein kinase activation and epithelial-mesenchymal transition in renal tubular epithelial cells. J Am Soc Nephrol 2005, 16:667-675.

18. Esnault S, Shen ZJ, Whitesel E, Malter JS: The peptidyl-prolyl isomerase Pin1 regulates granulocyte-macrophage colony-stimulating factor mRNA stability in T lymphocytes. J Immunol 2006, 177:6999-7006.

doi:10.1186/1755-1536-3-1

Cite this article as: Reese et al:: The Pin 1 inhibitor juglone attenuates kidney fibrogenesis via Pin 1-independent mechanisms in the unilateral ureteral occlusion model. Fibrogenesis \& Tissue Repair 2010 3:1.

Publish with Bio Med Central and every scientist can read your work free of charge

"BioMed Central will be the most significant development for disseminating the results of biomedical research in our lifetime. "

Sir Paul Nurse, Cancer Research UK

Your research papers will be:

- available free of charge to the entire biomedical community

- peer reviewed and published immediately upon acceptance

- cited in PubMed and archived on PubMed Central

- yours - you keep the copyright 